Z. klin. Chem. u. klin. Biochem.

7. Jg., S. $640-643$, November 1969

\title{
Vollautomatische Bestimmung des Blutzuckers nach der Hexokinasemethode
}

\author{
Von U. Harding unter Mitarbeit von G. Heinzed \\ Aus den Biocbemischen Forscbungslaboratorien der Firma Dr. Karl Thomae GmbH, Biberach/Riß
}

(Eingegangen am 29. Juli 1969)

Es wird ein Verfahren zur vollautomatischen Bestimmung der Glucose in Vollblut oder Serum mit dem Technicon AutoAnalyzer nach der Hexokinase-Methode beschrieben. Bei Einsatz von $20 \mu \mathrm{l}$-bzw. $50 \mu \mathrm{l}$-Blutproben können 70 Bestimmungen in der Stunde durchgeführt werden. Dabei werden je nach Registrierbereichseinstellung $1-5 \mathrm{mg} / 100 \mathrm{ml}$ Glucose einwandfrei erfaßt. Meßbereich, Meßgenauigkeit, Wiederfindungsrate, Vergleiche mit der manuellen Methode und der „Mikro-Glucose-Bestimmung“ am AutoAnalyzer sowie die Kosten der Analysen werden angegeben und diskutiert.

\section{A fully automated determination of blood sugar by the bexokinase method}

A completely automatic procedure, based on the hexokinase method, is described for the determination of glucose in whole blood or serum with the autoanalyser. 70 Determinations per hour can be made with blood samples of $20 \mu l$ or $50 \mu l$. Depending on the selected recording range, $1-5 \mathrm{mg} / 100 \mathrm{~m} /$ glucose is easily measured. The range of measurement, the accuracy, the recovery, a comparison with manual methods and with the "micro glucose determination" on the autoanalyser, and the cost are presented and discussed.

Die Bestimmung des Blutzuckers mit Hilfe von Hexokinase ${ }^{1}$ ) und Glucose-6-phosphatdehydrogenase wird übereinstimmend als die spezifischste und genaueste Methode bezeichnet (1-7). Ihre breite Anwendung bei klinisch-chemischen und biochemischen Routinemessungen scheiterte bisher an dem im Vergleich mit anderen Methoden sehr hohen Preis und am benötigten Zeitaufwand. Während kolorimetrische Blutzuckermessungen, z. B. mit Ferricyanid (8) oder o-Toluidin (9-11) und die enzymatische Bestimmung mit Glucoseoxydase (12-17) für das System des Technicon AutoAnalyzers beschrieben sind, fehlt dies für die Hexokinasemethode. Lediglich eine halbautomatische Mikrobestimmung von Glucose im Urin ist bekannt (18).

Wir möchten deshalb im folgenden eine vollautomatische Bestimmung der Glucose aus Vollblut oder Serum am Technicon AutoAnalyzer unter Verwendung der Boehringer Test Combination (UV-Test) und des EppendorfPhotometers vorschlagen, die sehr einfach und infolge der großen Verdünnungen der Reagenzien auch relativ billig durchführbar ist.

\section{Methodik}

\section{Prinzip}

Die zu messenden Blutproben werden mit $\mathrm{NaCl}$-Lösung verdünnt in den AutoAnalyzer gegeben und durch Dialyse gegen das Reaktionsgemisch in $\mathrm{NaCl}$ enteiweißt. Die dabei in das Enzymgemisch übertretende Glucose wird folgendermaßen umgesetzt:

$$
\begin{aligned}
& \text { ATP + Glucose } \frac{\text { Hexokinase }}{\mathrm{Mg}^{2+}, \mathrm{K}^{+}} \rightarrow \text { Glucose-6-phosphat + ADP; } \\
& \text { Glucose-6-phosphat } \frac{\text { Glucose-6-phosphat- }}{\text { dehydrogenasc }} \rightarrow \text { Gluconat-6-phosphat } \\
& +\mathrm{NADP} \text { dehydrogenase }+\mathrm{NADPH}_{2}
\end{aligned}
$$

1) Der Trivialname Hexokinase wird hier gebraucht für ATP: D-Hexose 6-Phospho-transferase (EC 2.7.1.1), Glucose-6-phosphatdehydrogenase für D-Glucose-6-phosphat: NADP Oxydoreduktase (EC 1.1.1.49), Glucosenxydase für $\beta$-D-Glucose: $\mathrm{O}_{2}$ Oxydoreduktase (EC 1.1.3.4) und Peroxydase für Donor: $\mathrm{H}_{2} \mathrm{O}_{2}$ Oxydoreduktase (EC 1.11.1.7).
Das $\mathrm{NADPH}_{2}$ ist der phosphorylierten Glucosemenge äquimolar und wird photometrisch bei $334 \mathrm{~nm}$ gemessen.

Reagenzien und Lösungen

Reagenzien

$\mathrm{NaCl}$ p. a. (Merck 6406)

Brij 35 (Technicon)

$\mathrm{D}(+)$ Glucose (Merck 8337)

Biochemica Test Combination Glucose TC-X, (UV-Test, TGAA 15994 bzw. TGAB 15931, Boehringer Mannheim)

Verdünnungslösung

0,9 proz. $\mathrm{NaCl}-\mathrm{Lösung}+0,5 \mathrm{~m} / / \mathrm{Brij}$.

\section{Reaktionsgemisch}

Der Inhalt der Fläschchen 1-3 der Test Combination TGAB 15931 wird unter Kühlung im Eisbad in einer braunen Flasche mit bidest. Wasser auf $425 \mathrm{ml}$ (bei TGAA 15994 auf $85 \mathrm{~m} /$ ), Fläschchen 4 unter gleichen Bedingungen mit $175 \mathrm{ml}$ (bzw. $35 \mathrm{ml}$ ) bidest. Wassex aufgefüllt. Um die Enzyme und Coenzyme vollständig zu überführen, sollten die Flaschen mit Wasser ausgespült werden. Nach gutem Durchschütteln werden die Gemische im Eisbad an den AutoAnalyzer angeschlossen. Sie müssen täglich neu angesetzt werden. Mit den $600 \mathrm{~m} /$ können je nach Einstellung der Probennehmer-Geschwindigkeit 360-480 Proben gemessen werden (mit $120 \mathrm{~m} / 70-90$ Proben).

\section{Glucosestandards}

Einwiegen von $\mathrm{D}(+)$ Glucose und Auffüllen mit bidest. Wasser zu folgenden Konzentrationen: 50,100 und $200 \mathrm{mg} / 100 \mathrm{~m} /$.

\section{Geräte}

Technicon AutoAnalyzer (Standardausführung) mit Probennehmer II (Nockenscheibe [1:1] für 60 oder 70 Proben/h), Propor tionierpumpe, Dialysator $\left(37^{\circ}\right)$, Doppelheizbad $\left(37^{\circ}\right)$ und TygonSchläuchen. Als Meßgerät dient das Eppendorf-Photometer 1101 M (Netheler und Hinz, Hamburg), Filter 334 nm, mit Transformationsstufe 1853 (Registrièrsbereichseinstellung $0-0,25$ ) und elektronischem Kompensationsschreiber 4412. Dazu wurde die Technicon Zylinder-Durchflußküvette $(15 \mathrm{~mm})$ 105-1475-15 vor den Eppendorf-Küvettenhalter 1155 zwischen Blende und Filter adaptiert (Abb.1). Falls pro Probe rẹgelmäßig $50 \mu l$ Blut zur Verfügung stehen, kann anstelle dieser Küvette ohne weiteres auch die $10 \mathrm{~mm}$-Eppendorf-Durchflußküvette verwendet werden. Zur Vermeidung des Apparatedrifts empfiehlt es sich, das Gerät 
Der zur Förderung der Forschung gestiftete

\section{HEINRICH-WIELAND-PREIS}

wird hiermit satzungsgemäß für das Jahr 1970 ausgeschrieben.

Der Preis, benannt nach dem 1957 verstorbenen Nobelpreisträger Professor Dr. Heinrich Wieland, ist für Arbeiten aus der Chemie, Biochemie und Physiologie der Fette und Lipoide sowie über deren ernährungsphysiologische und klinische Bedeutung ausgesetzt und wird jährlich verliehen.

Der HEINRICH-WIELAND-PREIS besteht aus einer „Heinrich-WielandPlakette", einem Geldbetrag in Höhe von 10000 DM, der Übernahme aller mit Vervielfältigung und Verteilung der ausgezeichneten Arbeit bis zu einer Auflage von 100 Exemplaren verbundenen Kosten.

Ein Kuratorium, dem zur Zeit die Herren

Prof. Dr. Werner Droese, Dortmund Prof. Dr.Werner Heimann, Karlsruhe Prof. Dr. Joachim Kühnau, Hamburg Prof.Dr.Dr.K. Lang, Bad Krotzingen Prof.Dr. Rudolf Pannhorst, Berlin Prof. Dr. Gotthard Schettler, Heidelberg Prof. Dr.Theodor Wieland, Heidelberg Prof. Dr.Viktor Wolf, Hamburg

Prof. Dr. Nepomuk Zöllner, München angehören, wird den Preisträger auswählen.

Einsendeberechtigt für die Verleihung des HEINRICH-WIELAND-PREISES für das Jahr 1970 sind Autoren von unveröffentlichten oder in den Jahren 1968 bis 1970 publizierten wissenschaftlichen Arbeiten. Der eingereichte Beitrag muß in deutscher, englischer oder französischer Sprache abgefaßt sein. Bei fremdsprachlichen Arbeiten ist eine ausführliche Zusammenfassung (etwa 10-15 Seiten) in deutscher Sprache erforderlich. Abhandlungen, die bereits mit einem anderen wissenschaftlichen Preis ausgezeichnet sind, können nicht prämiiert werden.

Einsendeschluß für die Verleihung im Jahre 1970 ist der 1. März 1970.

Die Arbeiten sind in einem Exemplar bis zu diesem Datum an folgende Anschrift einzusenden:

Kuratorium für die Verleihung des HEINRICH-WIELAND-PREISES Im Auftrag Prof. Dr. Alfons Fricker, 7501 Grötzingen, Ringelberghohl 12 a 


\section{Pfortaderhochdruck}

\section{und Eiweißstoffwechsel}

\section{Das große Rezeptbuch der Haut- und Körperpflegemittel}

Eine Einführung in die Praxis der Herstellung kosmetischer Erzeugnisse

Vierte, durchgesehene Auflage, herausgegeben von Paul Piep. 810 Seiten. Mit zahlreichen Tabellen und Formeln. Kunststoffeinband mit Schutzumschlag DM 58,-

Umfassende Sammlung erprobter Rezepte mit ausführlichen Arbeitsvorschriften aus vierzigjähriger Praxis, ergänzt durch wertvolle Rezepturen aus eigener Fabrikationstätigkeit des Bearbeiters, von Entwicklungslaboratorien und Forschungsstätten, unter besonderer Berücksichtigung der natürlichen, biologischen Kosmetik.

Aus dem Inhalt: Die biologischen Wirkstoffe (Wirkstoffe in kosmetischen Präparaten; Heil- und Giftpflanzen in der kosmetischen Praxis) - Die kosmetischen Rohstoffe (Alkohole; Chemikalien, Drogen, Grundstoffe und Hilfsmittel für die kosmetische Praxis; Fette und Öle; Fettalkohole; Fettsäuren; Wachse; Wässer; Farbstoffe; Konservierungsmittel; Parfümstoffe in kosmetischen Präparaten; Schädigungen der Haut) - Die Praxis der kosmetischen Fabrikation (Laboratorium und Fabrikationsräume; AerosolPraktikum; Antiperspirants; Desodorants; Azulen-Präparate; Enthaarungsmittel; Haarpflegemittel; Hautpflegemittel; Anti-Faltenöle und Hautöle; Feuchtigkeitspräparate; Gesichtspackungen; Massagemittel; Sommersprossenmittel; Sonnenschutz- und Bräunungsmittel mit Strahlenschutz; Sonnenbrandpuder; Waschungen und Badezusätze; Puder; Schönheitsmittel; Nagelpflegemittel; Mundund Zahnpflegemittel; Herren-Kosmetik; Intime Körperpflegemittel).

$\mathrm{Zu}$ beziehen durch Buchhandlungen im In- und Ausland, "andernfalls durch den Verlag.

Unseren Spezialprospekt übersenden wir Ihnen gern auf Anforderung.

Dr. Alfred Hüthig Verlag GmbH
Heidelberg

Mainz

Basel
Das vorliegende Buch ist für die Zukunft besonders wertvoll, da die Krankheiten der Leber mit der Hepatitis an der Spitze ihrer Infektionskrankheiten in allen Ländern zahlenmäßig ansteigen und daher mit einer $\mathrm{Zu}$ nahme der ,jetzt schon häufigen Leberzirrhose" und im Gefolge des damit zusammenhängenden Pfortaderhochdrucks mit vermehrt auftretenden lebensbedrohlichen Varizenblutungen $z u$ rechnen ist.

Das gut ausgestattete Buch enthält 49 Abbildungen und 56 Tabellen. Der Inhalt gliedert sich einschließlich der Einführung und Zusammenfassung in 9 Hauptkapitel. Angeschlossen sind eine ausführliche Literaturaufstellung und ein Sachverzeichnis.

Das Buch besitzt nicht nur einen speziellen Wert für die Klinik der Lebererkrankungen. Vielmehr ist es bei der heutigen zahlenmäßigen Verbreitung der Leberzirrhose für jeden Arzt von besonderer Wichtigkeit, den Inhalt dieser Arbeit zu kennen.

Rundschreiben des Verbandes der für Berufsgenossenschaften tätigen Ärrțe

Es werden die für die Indikation erforderlichen klinischen Untersucbungsmethoden eingebend geschildert sowie die Ergebnisse tierexperimenteller Forscbungen, die mit den an Menschen gefundenen nicht immer übereinstimmen.

Die operative Kontraindikationen werden genau erörtert.

Prof. Dr. Franke in Medizinische Neuerscbeinungen

Walter de Gruyter \& Co $\cdot$ Berlin 


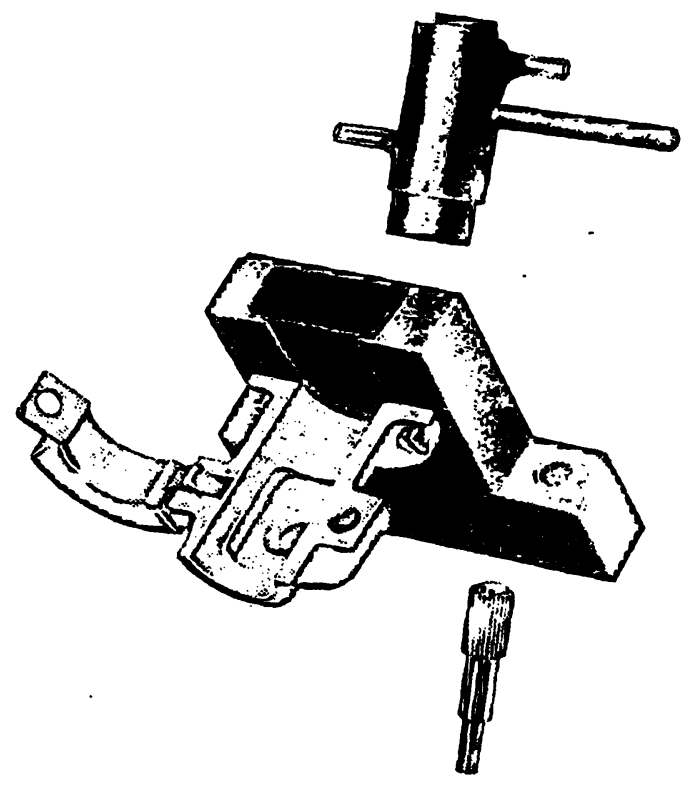

Abb. 1

Adaption der Technicon-Durchflußküvette $(15 \mathrm{~mm})$ an den Küvettenhalter des Eppendorf-Photometers. Oben: Durchflußküvette. Unten neuer Küvettenhalter

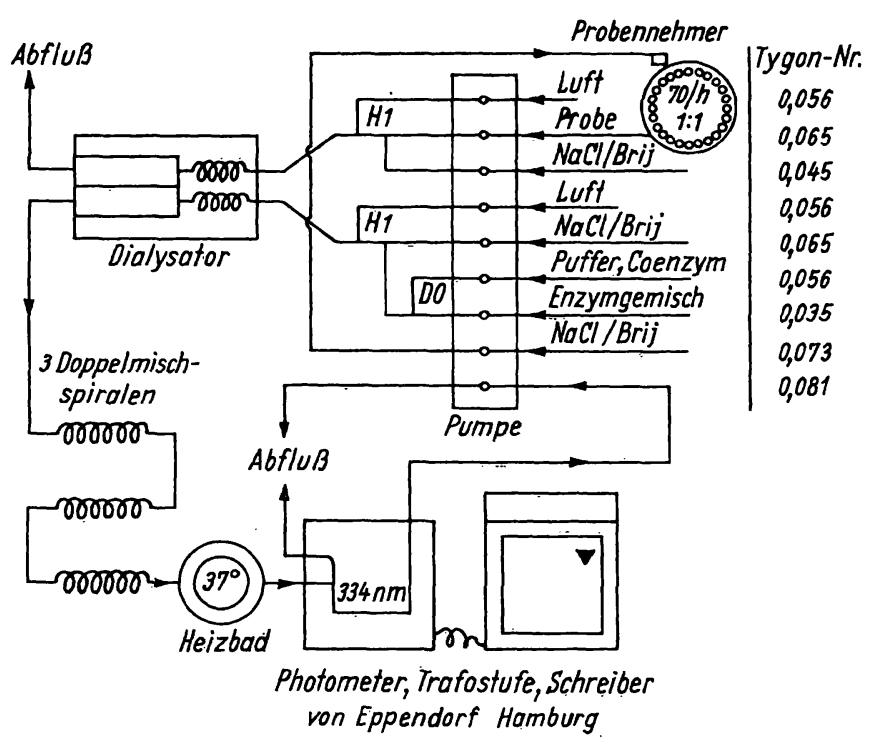

Abb. 2

Fließschema der Blutglucosebestimmung (Hexokinasemethode) am AutoAnalyzer. Kombination von Technicon-Probennehmer und Pumpe mit Eppendorf-Photometer und Schreiber. Die Weiten der Tygonschläuche in der Proportionierpumpe sind nach dem Technicon-Datenblatt für Schlauchgrößen angegeben. DO und $\mathrm{H}_{1}=$ Typ der benutzten Glasfittings auch über Nacht bei offenem Lichtweg eingeschaltet zu halten. Probenbecher beziehen wir von der Firma AS-Nunc (Algade 8, Roskilde, Dänemark, Bestell-Nr. 1151). Verdünnung der Blutproben erfolgt mit Citopipetten der Fa. E. Bühler, Tübingen, die Durchmischung mit Rührstab (3350) der Firma Eppendorf.

\section{Ausführung}

Aufbau und Arbeitsweise der AutoAnalyzer-Anlage crgeben sich aus Abbildung 2. Zur Inbetriebnahme wird die Apparatur zunächst 5 Min. mit dem Reaktionsgemisch durchspült und danach die Basislinie eingestellt (Schreiberausschlag 0\%).

$20 \mu l$ des zu untersuchenden Blutes bzw. der Standardlösungen werden in den Probenbechern mit $0,8 \mathrm{~m} /$ Verdünnungslösung versetzt (bei Verwendung von $50 \mu / \mathrm{mit} 1,0 \mathrm{~m} /$ ), gut gemischt und in den Probennehmer eingesetzt. Vor jeweils 40 Bestimmungen werden 2 Standardproben (50 oder $100 \mathrm{mg} / 100 \mathrm{~m} /$ ) mitgemessen. Die Entnahme am Probennehmer erfolgt mit einer Rate von 70 pro Std. Durch Dialyse gegen das Reaktionsgemisch wird die Probe enteiweißt und die Glucose mit den Coenzymen und Enzymen vereinigt. Nach Durchgang durch 3 Doppelmixspiralen und das Heizbad $\left(37^{\circ}\right)$ erfolgt die Messung des gebildeten $\mathrm{NADPH}_{2}$ bei $334 \mathrm{~nm}$.

\section{Charakteristik der Methode und Diskussion}

\section{Meßbereich}

Abbildung 3 zeigt, daß die Schreiberausschläge unter den gewählten Bedingungen bei den für die Blutmessungen wichtigen Konzentrationen streng proportional der Glucosemenge sind. Bei Verwendung von $20 \mu$-Proben und einer Registrierbereichs-Einstellung von $0-0,5$ sind Glucosekonzentrationen bis $500 \mathrm{mg} /$ $100 \mathrm{ml}$ meßbar, größere Mengen durch Verdünnung der Proben. Die kleinste meßbare Glucosemenge beträgt in diesem Falle $5-6 \mathrm{mg} / 100 \mathrm{ml}$, bei Veränderung des Registrierbereichs auf $0-0,25$ dagegen $2-3 \mathrm{mg} /$ $100 \mathrm{ml}$, wobei es sich bei der Messung solch kleiner Glucosemengen empfiehlt, 50-100 $\mu$-Proben zu verwenden. Dadurch wird eine Konzentration von $1 \mathrm{mg} /$ $100 \mathrm{ml}$ noch gut bestimmbar, abgesehen von der Verringerung des Pipettierfehlers, der dann bei $20 \mu l$ schon erheblich ist.

\section{Meßgenauigkeit}

Zur Überprüfung der Meßgenauigkeit wurde ein Gemisch aus Seren von 14 Ratten verwendet, mit dem insgesamt 9 Versuche mit je 5 Bestimmungen unter völlig gleichen Bedingungen durchgeführt wurden. Die Meßwerte, deren Mittelwerte und die resultierenden mittleren Fehler der Mittelwerte sind in Tabelle 1 wiedergegeben. Der Fehler beträgt durchschnittlich 0,4 bis $0,8 \mathrm{mg} / 100 \mathrm{ml}$. Wird eine noch größere Genauigkeit

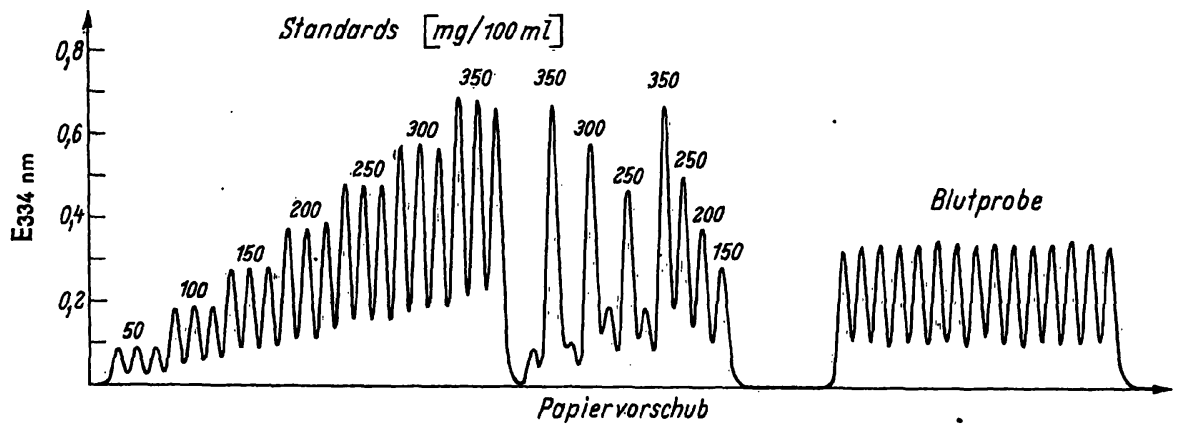

Abb. 3

AutoAnalyzer-Diagramm zum Nachweis der Beziehung zwischen Glucosekonzentration und Extinktion. Hexokinasemethode, $20 \mu l$-Standard oder-Blutproben, Registrierbereich $0-0,5$ Nockenscheibe $70 / \mathrm{h}$ (1:1). Zunächst Mehrfachmessung von Standards steigender Konzentration dann Einfachmessung von Standards stark unterschiedlicher Höhe und schließlich Mehrfachmessung einer Blutprobe 
Tab. 1

Prüfung der Meßgenauigkeit der Glucosebestimmung am Auto-Analyzer nach der Hexokinasemethode mit einem Gemisch aus 14 Rattenseren. 9 Versuche mit je 5 Messungen, Registrierbereich $0-0,25$, Nockenscheibe 70/h $1: 1, \bar{x}=$ Mittelwert, $\mathrm{sx}_{\mathbf{x}}=$ mittlere $20 \mu \mathrm{l}$

\begin{tabular}{ccccccccc}
\hline Versuch & & \multicolumn{3}{c}{$\mathrm{x}$} & & & $\overline{\mathbf{x}}$ & $\pm \mathrm{s} \overline{\mathbf{x}}$ \\
\hline 1 & 126 & 128 & 129 & 131 & 129 & 128,5 & 0,81 \\
2 & 127 & 131 & 129 & 130 & 130 & 129,5 & 0,67 \\
3 & 132 & 130 & 128 & 130 & 128 & 129,5 & 0,67 \\
4 & 128 & 130 & 131 & 128 & 127 & 129,0 & 0,74 \\
5 & 128 & 126 & 128 & 131 & 129 & 128,5 & 0,81 \\
6 & 128 & 127 & 127 & 127 & 130 & 128,0 & 0,59 \\
7 & 130 & 131 & 128 & 127 & 128 & 129,0 & 0,74 \\
8 & 128 & 128 & 128 & 128 & 130 & 128,0 & 0,39 \\
9 & 128 & 128 & 127 & 127 & 130 & 128,5 & 0,50 \\
\hline & & & & & Mittelwert: & 128,5 & \\
\hline
\end{tabular}

erforderlich, so empfehlen wir die Verwendung der Nockenscheibe 60/h $(1: 1)$. Wir haben auch hier mit obigem Sammelserum 5 Versuche mit je 5 Bestimmungen durchgeführt und hierbei einen durchschnittlichen mittleren Fehler der Mittelwerte von etwa $\pm 0,2-0,5 \mathrm{mg}$ / $100 \mathrm{~m} l$ erhalten.

Andere Blutbestandteile stören die Bestimmung nicht, wie Messungen von Blut bzw. Blut + NADP ohne Enzymzugabe zeigten.

\section{Wiederfindungsrate}

Von einem Mischserum aus Blut von 10 Ratten wurden $4 \mathrm{~m} l \mathrm{mit} 160 \mathrm{~m} l \mathrm{NaCl}$-Lösung verdünnt. Beim Kontrollversuch ohne Glucosezusatz wurden $\mathrm{zu} 0,8 \mathrm{ml}$ dieser Mischung $20 \mu \mathrm{l}$ Wasser pipettiert, bei den anderen $20 \mu \mathrm{l}$ Proben von Glucosestandards steigender Konzentration, deren Gehalt vorher überprüft worden war. Die Bestimmung wurde mit Nockenscheibe $70 / \mathrm{h}(1: 1)$ durchgeführt. Tabelle 2 zeigt, daß die zugesetzte Glucose zu $100 \%$ wiedergefunden wurde.

Tab. 2

Messung der Wiederfindungsrate bei Blutzuckerbestimmungen mit dem AutoAnalyzer (Hexokinasemethode) durch Zugabe verschiedener em Gemisch aus Blut von 10 Ratten. Die Angaben sind Mittelwerte aus je 5 Bestimmungen, $s \bar{x}=$ mittlere Feh-

\begin{tabular}{|c|c|c|c|}
\hline $\begin{array}{l}\text { Glucosek } \\
\text { Blutprobe }\end{array}$ & $\begin{array}{l}\text { entration in } \\
\text { zugesetzte } \\
\text { Glucose }\end{array}$ & $\begin{array}{l}1100 \mathrm{ml} \pm s_{\bar{x}} \\
\text { Blut }+ \text { Glucose }\end{array}$ & $\begin{array}{l}\% \text { Wieder- } \\
\text { findung }\end{array}$ \\
\hline $73 \pm 0,58$ & - & - & 100 \\
\hline $73 \pm 0,58$ & 50 & $124,5 \pm 0,60$ & 101 \\
\hline $73 \pm 0,58$ & 100 & $172,5 \pm 0,68$ & 99,5 \\
\hline $73 \pm 0,58$ & 150 & $225,5 \pm 0,51$ & 101 \\
\hline $73 \pm 0,58$ & 200 & $276,0 \pm 1,10$ & 101 \\
\hline $73 \pm 0,58$ & 250 & $322,5 \pm 0,51$ & 99,8 \\
\hline $73 \pm 0,58$ & 300 & $374,0 \pm 0,55$ & 100,8 \\
\hline $73 \pm 0,58$ & 350 & $423,0 \pm 0,32$ & 99,9 \\
\hline $73 \pm 0,58$ & 400 & $472,5 \pm 0,87$ & 99,8 \\
\hline
\end{tabular}

Vergleich quischen manueller und automatischer Messung Wir haben die manuelle und automatische Glúcosebestimmung mit Hexokinase mit der automatischen Mikro-Glucose-Bestimmung $(70 / \mathrm{h}, 2: 1)$ verglichen. Dazu wurde im Serum von 14 normalen Ratten in jeweils 5 Messungen der Glucosegehalt nebst mittlerem Fehler des Mittelwertes bestimmt und die Abweichungen bei den beiden anderen Methoden im Vergleich zur
Tab. 3

Vergleich der Meßdaten bei Blutzuckerbestimmungen mit der Hexokinase- und Mikromethode am AutoAnalyzer und der Handmethode mit Hexokinase. Verschiedene Seren normaler Ratten, 14 Versuche mit Mittelwerten aus je 5 Messungen und mittleren Fehlern der Mit Mittelwerten aus $(\mathrm{s})$ in $100 \mathrm{ml}$. Die Abweichungen beziehen sich auf die Hexokinasemethode am AutoAnalyzer

\begin{tabular}{|c|c|c|c|c|c|}
\hline Tier & $\begin{array}{l}\text { AutoAnalyzer } \\
\text { (Hexokinase) }\end{array}$ & $\begin{array}{l}\text { AutoAn } \\
\text { (Mikrom }\end{array}$ & $\begin{array}{l}\text { alyzer } \\
\text { thode) }\end{array}$ & $\underset{\text { (Hex }}{\text { Hand }}$ & \\
\hline & $\overline{\mathbf{x}} \pm s_{\bar{x}}$ & $\overline{\mathbf{x}} \pm \mathbf{s} \overline{\mathbf{x}}$ & $\begin{array}{l}\% \text { Ab- } \\
\text { weichung }\end{array}$ & $\overline{\mathbf{x}} \pm \mathrm{s} \overline{\mathbf{x}}$ & $\begin{array}{l}\% \text { Ab- } \\
\text { weichung }\end{array}$ \\
\hline 1 & $72 \pm 0,37$ & $77 \pm 0,90$ & $+7,5$ & $72 \pm 1,33$ & $+0,7$ \\
\hline 2 & $128 \pm 0,58$ & $129 \pm 0,55$ & $+0,55$ & $127 \pm 1,05$ & $-0,5$ \\
\hline 3 & $130 \pm 0,58$ & $131 \pm 0,32$ & $+0,6$ & $130 \pm 1,40$ & \pm 0 \\
\hline 4 & $145 \pm 1,24$ & $146 \pm 0,25$ & $+0,6$ & $144 \pm 1,35$ & $-0,7$ \\
\hline 5 & $130 \pm 1,36$ & $132 \pm 0,22$ & $+1,3$ & $129 \pm 0,81$ & $-0,65$ \\
\hline 6 & $143 \pm 0,68$ & $147 \pm 0,20$ & $+2,3$ & $143 \pm 0,36$ & \pm 0 \\
\hline 7 & $138 \pm 0,66$ & $143 \pm 0,30$ & $+3,6$ & $\cdot 138 \pm 2,48$ & $+0,1$ \\
\hline 8 & $130 \pm 0,51$ & $133 \pm 0,43$ & $+2,3$ & $130 \pm 0,32$ & \pm 0 \\
\hline 9 & $148 \pm 0,49$ & $150 \pm 0,20$ & $+1,35$ & $147 \pm 1,12$ & $+0,5$ \\
\hline 10 & $139 \pm 0,51$ & $141 \pm 0,25$ & $+1,9$ & $139 \pm 0,36$ & $+0,1$ \\
\hline 11 & $133 \pm 0,37$ & $137 \pm 0,55$ & $+2,8$ & $131 \pm 1,61$ & $-1,05$ \\
\hline 12 & $125 \pm 0,37$ & $131 \pm 0,76$ & $+5,0$ & $125 \pm 0,73$ & \pm 0 \\
\hline 13 & $127 \pm 0,93$ & $132 \pm 1,17$ & $+3,7$ & $126 \pm 1,99$ & $-1,0$ \\
\hline 14 & $132 \pm 0,51$ & $134 \pm 0,37$ & $+2,0$ & $133 \pm 1,12$ & $+0,8$ \\
\hline
\end{tabular}

automatischen Hexokinase-Methode berechnet (Tab. 3). Es zeigt sich, daß die Mittelwerte beider Bestimmungen mit Hexokinase sehr gut übereinstimmen (durchschnittliche Abweichung hier 0,4\%). Die $\mathrm{S}_{\overline{\mathbf{x}}}$ sind bei der Handmethode wesentlich größer, was wohl vor allem auf die unterschiedliche Dauer der Endpunkteinstellung und auf Pipettierfehler zurückzuführen ist.

Die $\mathrm{S}_{\overline{\mathbf{x}}}$-Werte bei der Mikro-Glucose-Messung sind ebenfalls sehr niedrig. Man muß aber berücksichtigen, $\mathrm{da} \beta$ mit dieser Methode ja nicht der "wahre" Glucosewert ermittelt wird. Die Abweichungen der Mittelwerte von den Hexokinase-Werten sind daher natürlich wesentlich höher (bis zu 7,5\%). Dennoch sind die erhaltenen Abweichungen bei weitem kleiner als die in der Literatur beschriebenen $(2,3,6)$. Dies ist auf die Verwendung von Normalserum anstelle von pathologischen Seren bei unseren Versuchen zurückzuführen.

\section{Kosten des Verfabrens}

Die manuelle Bestimmung der Glucose mit dem Hexokinase-Verfahren ist weitaus die teuerste, weshalb sie bisher keine Verwendung bei Routinemessungen finden konnte. Bei der hier beschriebenen automatischen Methode verringert sich der Preis durch die kleine Konzentration der zur Messung einer Probe benötigten Coenzyme und Enzyme stark. Bei der Verwendung der Nockenscheibe $70 / \mathrm{h}(1: 1)$ können unter Berücksichtigung von Anlaufzeit und Messung von Standardproben mit $600 \mathrm{~m} /$ Reaktionsgemisch (=1 Boehringer Testpackung) 400 Proben gemessen werden. Damit ergibt sich ohne Erstanschaffung und Personalkosten ein Preis von 0,16 DM, bei der manuellen Bestimmung mit der Boehringer Testpackung dagegen 0,64 DM, wobei zu berücksichtigen ist, daß hier die zusätzlichen Personalkosten wesentlich höher sind als bei Verwendung des AutoAnalyzers. Damit liegen die Kosten in der gleichen GröBenordnung wie bei den automatischen Bestimmungen der Glucose mit o-Toluidin oder Glucoseoxydase/Peroxydase $(10,13,15)$. 
Die vorgeschlagene automatische Methode vereinigt also die Vorteile der sehr spezifischen enzymatischen Glucosebestimmung mit einer sehr einfachen technischen Durchführung der Messung : Blut kann direkt verwendet werden, es ist keine vorherige Enteiweißung nötig und die Benutzung der Boehringer Testpackung erfordert keine zeitraubende Zubereitung der Reagenzien. Damit werden weitere Fehlerquellen ausgeschaltet. Weiter ist hervorzuheben, daß die benötigten Blutmengen sehr klein sind, mit 70 Proben/h eine hohe Meßkapazität zur Verfügung steht und der Preis pro Bestimmung erheblich niedriger ist als bei der manuellen Methode.

Fräulein X. LuFT und Fräulein J. Meste sind wir für technische Mitarbeit, Herrn H. SchwarTz für die Anfertigung des Küvettenhalters zu Dank verpflichtet.

\section{Literatur}

1. Schmidt, F. H., Internist, 4, 554 (1963). - 2. SchNoOR, O., A. Delbrüick und W. Barthelmat, Med. Klin. 59, 1230 (1964). 3. Förster, H., H. Mehnert und K. Stuhlfauth, Münch. Med. Wschr. 107, 1441 (1965). - 4. Reinauer, H. und S. HollmanN, in D-Glucose und Verwandte Verbindungen in Medizin und Biologie, S. 71, Hrsg. von H. BARTELHEIMER, W. HEYde und W. Thorn, F. Enke Verlag Stuttgart (1966). - 5. Breuer, H., ibid., S. 658. - 6. Lorenz, L. und C. LüdemanN, Dtsch. Med. J. 18, 420 (1967). - 7. Wüst, H., Therapiewoche 26/67, S. 879 (1967). - 8. Grady, H. J. und M. A. Lamar, Clin. Chem. New York 5, 542 (1959). - 9. ZENDER, R., Clin. chimica Acta Amster- dam, 8, 351 (1963). - 10. LeYBold, K., diese Z. 6, 51 (1968). 11. Scrürz, W., Ärztl. Lab. 14, 500 (1968). - 12. HiLL, J. B. und G. KessLer, J. Laborat. Clin. Med. S. Louis 57, 970 (1961). - 13. KOHLER, K., in „Automation in der Analytischen Chemie“, Technicon Symposia, 1963, Sonderdruck 256. - 14. Gretchell, G., G. R. Kingsley und R. R. Schaffert, Clin. Chem. New York 10, 540 (1964). - 15. Robin, M. und A. SAIFER, Clin. Chem. New York 11, 840 (1965). - 16. KAweran, E., diese Z. 4, 224 (1966). 17. Tammes, A. R. und C. D. Nordshow, Amer. J. Clin. Path. 49, 1613 (1968). - 18. Schersten, B. und G. Tibbling, Clin. Chem. New York 14, 243 (1968).
Dr. Uwe Harding Biochemische Forschungslaboratorien der Fa. Dr. Kiarl Thomae GmbH 795 Biberach an der Riss 